\title{
Dangling bond spins controlling recombination dynamics of excitons in colloidal nanocrystals and nanoplatelets
}

\author{
(C) A.V. Rodina ${ }^{1}$, A.A. Golovatenko ${ }^{1}$, E.V. Shornikova ${ }^{2,3}$, D.R. Yakovlev ${ }^{1,2}$, Al.L. Efros ${ }^{4}$ \\ ${ }^{1}$ loffe Institute, \\ 194021 St. Petersburg, Russia \\ ${ }^{2}$ Experimentelle Physik 2, Technische Universität Dortmund, \\ 44227 Dortmund, Germany \\ ${ }^{3}$ Rzhanov Institute of Semiconductor Physics, Siberian Branch of Russian Academy of Sciences, \\ 630090 Novosibirsk, Russia \\ ${ }^{4}$ Naval Research Laboratory, \\ Washington DC 20375, USA \\ E-mail: Anna.rodina@mail.ioffe.ru
}

We show theoretically that spins of dangling bonds (DBS) at surface of colloidal nanostructures can be responsible for the dark exciton radiative recombination governing low temperature photoluminecence. The DBS-assisted dark exciton recombination is efficient when all spins are randomly oriented. The DBS polarization caused by external magnetic field or by the formation of the dangling bond magnetic polaron (DBMP) at low temperatures suppresses the DBS-assisted recombination. The temperature or magnetic field control on the DBS-polarization allows to detect experimentally the presence of DBS and DBS-assisted recombination in CdSe nanocrystals and $2 D$ nanoplatelets.

\section{Acknowledgement}

This work has been supported in part by the Government of Russia (project number 14.Z50.31.0021, leading scientist M.Bayer), by the RFBR (Grant No 17-02-01063), and by the Deutsche Forschungsgemeinschaft in the frame of ICRC TRR 160. 